breastfeeding and the lack of antiretroviral therapy during pregnancy.

\section{P3.78 LIPODYSTROPHY PREVALENCE AMONG PEOPLE LIVING WITH HIV IN SOUTHERN OF BRAZIL}

${ }^{1} \mathrm{M}$ Almeida da Silva, ${ }^{3} \mathrm{CEM}$ Marcon, ${ }^{4} \mathrm{RF}$ Sene, ${ }^{2} \mathrm{~F}$ Schuelter-Trevisol. ${ }^{1}$ Postgraduate Program in Health Sciences at University of Southern Santa Catarina, Brazil, ${ }^{2}$ Clinical Research Centre at Hospital Nossa Senhora da Conceição, Brazil

\subsection{6/sextrans-2017-053264.313}

Introduction HIV-associated lipodystrophy syndrome is a major adverse effect of highly active antiretroviral therapy (HAART), although it also occurs among people living with HIV who do not receive any pharmacological treatment. Lipodystrophy diminishes patients' quality of life and may hinder treatment compliance or lead to its abandonment.

Methods Cross-sectional study conducted from October 2015 to March 2016. A sample was recruited from individuals living with HIV who attended an outpatient clinic in Tubarão, state of Santa Catarina, Brazil. We collected information on demographics, lifestyle, HIV infection, and clinical aspects. Lipodystrophy was diagnosed through patient self-report associated with anthropometry.

Results We surveyed 405 patients (most were white men; mean age 43.7 years). The mean duration of HIV infection was 74.6 months, and $90.1 \%$ of the respondents were taking antiretroviral therapy. The prevalence of lipodystrophy was $34.2 \%$, of whom $43 \%$ had lipoatrophy, 52\% had lipohypertrophy, and $5 \%$ had a mixed form. There was a statistical association between the presence lipodystrophy and female gender $[\mathrm{OR}=1.77$ (95\% CI 1.35 to 2.32$)$ ] and the duration of HIV infection ( $[\mathrm{OR}=1.00$ (95\% CI 1.00 to 1.04$)]$.

Conclusion Lipodystrophy was prevalent in more than onethird of the surveyed subjects, which is a warning signal. Lipodystrophy affects quality of life and adherence to treatment, and may cause cardiovascular damage in this population.

\section{P3.79 BARRIERS FOR SYPHILIS SCREENING IN BOLIVIA}

${ }^{1}$ Tinajeros Freddy, ${ }^{2} \mathrm{R}$ Revollo, ${ }^{3} \mathrm{~L}$ Rey Ares, ${ }^{4} \mathrm{~V}$ Elias, ${ }^{4} \mathrm{~L}$ Reveiz. ${ }^{1} \mathrm{STI}$ and HIV Independent Consultant; ${ }^{2}$ Ministry of Health Bolivia; ${ }^{3}$ Research of Institute for Clinical and Sanitary Effectivity, Bolivia; ${ }^{4}$ Pan-American Health Organisation

\subsection{6/sextrans-2017-053264.314}

Introduction Syphilis is a global problem, with an estimated incidence of 12 million people infected each year and is a public health problem in Bolivia. This can result in fetal death, perinatal death, or severe neonatal infections. However, simple and cost-effective options for screening and treatment during pregnancy can reduce these complications.

Methods For the present study, mixed methods (qualitative and quantitative) were used, however the quantitative results are presented in the summary. The data were collected through a review of the prenatal control clinical records, from which data such as syphilis test results, results records, and treatment in the perinatal history were extracted. The data extracted from the medical records of the 8 health centres of the Los Andes Network were input into an Excel database and analysis was performed using Epi Info 7.

Results Of 294 clinical records reviewed, we observed that on average, $55.4 \%$ of patients had syphilis results attached to their clinical histories. The lowest percentage in any centre was $13.3 \%$ and the highest was $62.1 \%$. The percentages for each centre were: Alto Lima III 61.7\%, Alto Lima IV 45.8\%, Ambulatory Reference Centre (ARC) 61.1\%, German Busch 62.1\%, Huayna Potosí 67.3\%, Puerto Mejillones 40.0\%, Santa Rosa de Lima 13.3\% and Villa Ingenio 51.1\%. Significant differences $(p<0.05)$ were observed among the establishments of the first level of complexity without laboratory $(46.8 \%$, 95\% CI: 37.9-55.3) and the second level centres with laboratory $(63.5 \%, 95 \%$ CI $49.7-74.5)$. This suggests that the syphilis test is more likely to be performed in the second level than in the first level and that the results are recorded in the clinical records, mainly on the perinatal card.

Conclusion Failure to record results in the medical records could result in a pregnant woman being screened for syphilis without results and with the consequent risk that if she gives birth in a different health centre, she may not receive adequate treatment or necessary follow-up to the newborn. The sensitisation and monitoring to be implemented by the health personnel in each centre and a deeper discussion on the subject of syphilis can become catalysts for the health system. The sharing of the results of the study could allow the implementation of corrective measures to improve the monitoring of syphilis screening.

\section{P3.80 ANALYSIS OF THE CLINICAL PROFILE AND RESULTS OF TUBERCULOSIS CASES TREATMENT IN PEOPLE LIVING WITH HIVIAIDS}

Gabriela Tavares Magnabosco, Rubia Laine de Paula Andrade, Tiemi Arakawa, Maria Eugenia Firmino Brunello, Laura Terenciani Campoy, Livia Maria Lopes, Aline Aparecida Monroe, Tereza Cristina Scatena Villa. School of Nursing of Ribeirao Preto/ University of Sao Paulo, Ribeirao Preto - SP, Brazil

\subsection{6/sextrans-2017-053264.315}

Introduction Tuberculosis (TB) even though it is a viable cure is still recognised as a current and persistent public health problem aggravated by the rise in HIV/AIDS. The association between HIV infection and TB increases the probability of death of individuals and influences the control of both, challenging the practice of care and health policies.

Methods This was a descriptive, quantitative-type survey, aimed to analyse the clinical profile and results of TB treatment cases in people living with HIV/AIDS in Ribeirão Preto/ Brazil in the years 2010 to 2014 Patients in the penitentiary system, under the age of 18 and who had changes in diagnosis or transfer were excluded. TB/WEB information system was used for data collection. Descriptive statistical techniques were used for data analysis.

Results There were 224 cases of TB/HIV of which $71 \%$ were men and 29\% were women; $94.2 \%$ were diagnosed with AIDS and 5.8\% were HIV-infected. The clinical form of pulmonary TB was prevalent (64.7\%), followed by extrapulmonary $(23.2 \%)$ and pulmonary + extrapulmonary $(12.1 \%)$. Regarding the clinical profile of $\mathrm{TB}, 73.7 \%$ were new cases, $14.7 \%$ relapsed and $11.6 \%$ were re-treatment due to abandonment. Regarding the associated comorbidities, there were two cases with diabetes mellitus, $15.6 \%$ alcoholism, $15.6 \%$ drug addiction and $3.1 \%$ smoking. $74.6 \%$ of the cases had to be hospitalised at some point. Regarding the result, the cure rate was $57.2 \%, 16.5 \%$ of the abandonment and $26.3 \%$ of death. The clinical characteristics of the subjects follow the parameter of the general population (the highest prevalence among men, 
whites and economically active age group), except for the treatment result (the cure rate for coinfected patients is predominant, Which does not reflect the country profile, however, the death rate remains an alarming situation, as well as the hospitalisation rate for associated complications).

Conclusion The interaction of diseases requires the need for coordinated work developed by TB and HIV/AIDS programs to reduce the burden of both diseases and promote more favourable outcomes.

\section{P3.81 LATIN AMERICA PROFICIENCY TESTING PROGRAM FOR DIAGNOSIS AND ANTIMICROBIAL SUSCEPTIBILITY OF NEISSERIA GONORRHOEAE}

${ }^{1} \mathrm{P}$ Galarza, ${ }^{1} \mathrm{R}$ Gianecini, ${ }^{1} \mathrm{C}$ Oviedo, ${ }^{1} \mathrm{~K}$ Silva, ${ }^{2} \mathrm{P}$ Ramon Pardo, ${ }^{2} \mathrm{M}$ Ghidinelli, ${ }^{2} \mathrm{M}$ Galas and ${ }^{3}$ NGQCP Participants. ${ }^{1}$ Instituto Nacional de Enfermedades Infecciosas (INEI) - ANLIS. Ciudad Autonoma de Buenos Aires. Argentina; ${ }^{2}$ Pan-American Health Organisation (PAHO). World Health Organisation (WHO); ${ }^{3}$ Latin American Quality Control Comparison Program in Diagnostic and Antimicrobial Susceptibility Testing for Neisseria gonorrhoeae

\subsection{6/sextrans-2017-053264.316}

Introduction Since 2013 an ongoing international inter-laboratory quality control program (NGQCP) has analysed the capability of 16 national reference laboratories to diagnose and perform antimicrobial susceptibility testing (AST) for Neisseria gonorrhoeae in Latin America. The program is supported by PAHO/WHO for the GASP-RELAVRA network and coordinated by the Sexually Transmitted Disease Reference Laboratory (LC) - INEI from Argentina. Results of the 2016 fourth round of the NGQCP are presented.

Methods A panel with 5 isolates belong to Neisseriaceae family, is envoyed once by year. A questionnaire is attached to collect information from each codified laboratory (lab). The 2008 WHO panel and ATCC 49226 reference strains were distributed. NGQCP evaluate: presumptive and confirmatory identification and, AST by disk diffusion and Minimum inhibitory concentrations (MICs) determination by either agar dilution or Etest methods. MIC interpretations were based on the criteria of the Clinical Laboratory Standards Institute (CLSI).

Results Fifteen labs recovered isolates. Complete conventional presumptive and confirmatory identification was made by $73 \%$ and $87 \%$ of labs, respectively. Only 3 labs introduced a different confirmatory methodology. The $97.3 \%$ (72/74) of diagnostics were included in the category genus and species correct. Three (3/15) labs not realised $\beta$-lactamase detection. Disk diffusion assessment showed 31 minor, 2 major and 3 very major discrepancies. The overall agreement of MIC results (MICs $\pm 1 \log 2$ dilutions) between 8 labs, including the LC, compared to the modal MICs was 90\% (277/307). Percentage agreements by antibiotics was: penicillin 83\% (49/59); tetracycline 89\% (64/72); ciprofloxacin 93\% (53/57); ceftriaxone $90 \%$ (53/59); cefixime $88 \%(7 / 8)$ and azithromycin 98\% (51/ 52)

Conclusion NGQCP is an essential pillar for an effective surveillance program in order to ensure that their data are reliable. We expect more labs in the Region realise MIC determination by agar dilution methods and improve level of concordance for AST.

\section{P3.82 AN UPWARD TREND IN OCULAR SYPHILIS CASES IN BRITISH COLUMBIA, CANADA, 2013-2016: A DESCRIPTIVE ANALYSIS}

${ }^{1} \mathrm{G}$ Shumilak, ${ }^{2,3}$ J Wong, ${ }^{2} \mathrm{~B}$ Arnold, ${ }^{2,3} \mathrm{M}$ Gilbert, ${ }^{2} \mathrm{C}$ Lukac, ${ }^{2} \mathrm{~S}$ Makaroff, ${ }^{2} \mathrm{C}$ Montgomery, ${ }^{2,4}{ }^{1} \mathrm{D}$ Moore, ${ }^{2} \mathrm{C}$ Prescott, ${ }^{1,2} \mathrm{~T}$ Grennan. ${ }^{1}$ Division of Infectious Diseases, University of British Columbia, Vancouver, Canada; ${ }^{2} B C$ Centre for Disease Control, Vancouver, Canada; ${ }^{3}$ School of Population and Public Health, University of British Columbia, Vancouver, Canada; ${ }^{4} B C$ Centre for Excellence in HIVIAIDS, Vancouver, Canada

\subsection{6/sextrans-2017-053264.317}

Introduction HIV-positive individuals are generally considered higher risk for early and more serious neurologic complications related to syphilis. In 2014-2015, clusters of ocular syphilis cases were reported in the US. Simultaneously, the Canadian province of British Columbia (BC) saw a dramatic rise in infectious syphilis cases by $40 \%$. Here, we describe ocular syphilis cases diagnosed in BC.

Methods All neurosyphilis cases diagnosed in BC since 2013 were reviewed to identify ocular cases. Ocular syphilis was defined as having signs/symptoms of ocular disease (e.g. uveitis, blurred vision) and syphilis of any stage, as defined by the Centres for Disease Control and Prevention.

Results Between January $1^{\text {st }}, 2013$ and October 31 $1^{\text {st }}, 2016,35$ cases of ocular syphilis were recorded in BC. Most were male $(32 / 35 ; 91.4 \%)$ and identified as white $(20 / 35 ; 57.1 \%)$. The mean age was 49.7 years. A majority $(18 / 35$; 51.4\%) were living with HIV. The most frequent ophthalmologic diagnoses were uveitis (41.9\%), optic neuritis (12.9\%), and retinitis (9.7\%). Twenty-three cases had lumbar puncture data available: $13(56.5 \%)$ had elevated cerebrospinal fluid (CSF) protein, 15 (65.2\%) had elevated CSF cell count, and 6 (26.1\%) had positive CSF VDRL. As a proportion of all syphilis cases, ocular syphilis accounted for $0.80 \%$ of all cases during the 2013-2015 period, versus $1.54 \%$ for $2016(p=0.05)$. Stratified by HIV serostatus, there was a significant increase in the proportion of ocular syphilis cases in those living with HIV between the 2013-2015 and 2016 time periods $(1.17 \%$ vs. $3.21 \%, \mathrm{p}=0.03)$.

Conclusion Paralleling trends observed in some US jurisdictions, BC is experiencing an increase in ocular syphilis cases, and an increasing proportion of syphilis cases in those living with HIV are being diagnosed with ocular findings. These results further highlight the importance of continuing efforts to respond to the syphilis epidemic, and focused screening for ocular symptoms, particularly in those at highest risk.

\section{P3.83 ANTIMICROBIAL RESISTANCE OF UREAPLASMA UREALYTICUM AND MYCOPLASMA HOMINIS RESEARCHED AT A REFERENCE CENTRE IN SALVADOR, BAHIA}

${ }^{1} \mathrm{GC}$ Souza, ${ }^{2}$ E Xavier-Souza, ${ }^{2} \mathrm{MS}$ Timbó, ${ }^{1} \mathrm{~V}$ Cunha, ${ }^{1,3} \mathrm{AG}$ Travassos. ${ }^{1}$ Universidade do Estado da Bahia, Brazil; '2Universidade Federal da Bahia, Brazil; ${ }^{3}$ Centro Estadual Especializado em Diagnóstico, Assistência e Pesquisa (CEDAP), Brazil

\subsection{6/sextrans-2017-053264.318}

Introduction: Ureaplasma urealyticum (UU) and Mycoplasma hominis $(\mathrm{MH})$ are potentially pathogenic organisms commonly found in the urogenital tract, with colonisation rates up to $80 \%$ and $40 \%$ worldwide, respectively. The aim of this study was to estimate antimicrobial resistance by $\mathrm{UU}$ and $\mathrm{MH}$, and 